\title{
Correlaçáo entre Assistência do Cuidador e Desempenho Funcional em Crianças com Paralisia Cerebral
}

\author{
Correlation Between Caregiver Assistance and Functional Performance In Children with Cerebral Palsy \\ Jackeline Oliveira do Vale Cunha ${ }^{1}$, Geovana Sôffa Rézio
Cibelle Kayenne Martins Roberto Formiga
}

\section{RESUMO}

Objetivo. Este estudo teve o objetivo de identificar a correlação entre nível de assistência fornecido pelo cuidador e o desempenho funcional em crianças com Paralisia Cerebral (PC). Método. Doze (12) crianças com PC, nível I e II do GMFCS (Gross Motor Function Classification System), com idade média de 3 anos e 2 meses, frequentadoras do Setor Clínico da Associação Pestalozzi de Goiânia foram avaliadas através do Inventário de Avaliação Pediátrica de Incapacidade (PEDI - Pediatric Evaluation Disability Inventory). Resultados. Análise da correlação entre as partes de Habilidades Funcionais e Auxílio do Cuidador do PEDI indicou relação linear positiva significativa entre a assistência fornecida pelo cuidador e o desempenho funcional das crianças tanto na área de autocuidado $(\mathrm{r}=0,87 ; \mathrm{p}<0,001)$ quanto na mobilidade $(\mathrm{r}=0,78 ; \mathrm{p}=0,003)$. A correlação também se apresentou significativa no nível de independência da criança quanto à mobilidade e o desempenho da mesma nas atividades de autocuidado $(r=0,61$; $\mathrm{p}=0,033$ ). Conclusáo. Ressalta-se a necessidade de envolvimento dos cuidadores nos tratamentos e atividades direcionadas às crianças com Paralisia Cerebral para que estes apoiem a autonomia nas atividades funcionais de mobilidade e autocuidado de sua criança.

Unitermos. Paralisia Cerebral, Assistência do Cuidador, Desempenho Funcional.

Citaçáo. Cunha JOV, Rézio GS, Formiga CKMR. Correlação entre Assistência do Cuidador e Desempenho Funcional em Crianças com Paralisia Cerebral.

\begin{abstract}
Objective. This study aimed to identify the correlation between level of care provided by caregivers and functional performance in children with cerebral palsy (CP). Method. Twelve (12) children with CP, level I and II of the GMFCS (Gross Motor Function Classification System) with a mean age of 3 years and 2 months, attended the Clinical Section of the Association of Pestalozzi Goiânia were evaluated using the Pediatric Evaluation Disability Inventory (PEDI). Results. Correlation analysis of the Functional Skills and Caregiver Assistance of the PEDI showed significant positive linear relationship between the caregiver and the care provided by the functional performance of children both in self-care $(\mathrm{r}=0.87, \mathrm{p}<0.001)$ and mobility $(\mathrm{r}=0.78$, $\mathrm{p}=0,003$ ). The correlation also showed a significant level of independence as the child's mobility and its performance in self-care activities $(r=0.61, p=0,033)$. Conclusion. We stress the need to involve carers in treatment and activities targeted to children with cerebral palsy so that they support the activities of functional autonomy in mobility and self-care of their child.
\end{abstract}

Keywords. Cerebral Palsy, Caregiver Assistance, Functional Performance.

Citation. Cunha JOV, Rézio GS, Formiga CKMR. Correlation Between Caregiver Assistance and Functional Performance In Children with Cerebral Palsy.
Trabalho realizado na Associaçáo Pestalozzi de Goiânia, Unidade Renascer, Goiânia, Goiás, Brasil.

1.Terapeuta Ocupacional, Especialista em Intervenção em Neuropediatria, Universidade Federal de São Carlos (UFSCar), Goiânia-GO, Brasil.

2.Fisioterapeuta, Mestranda em Ciências Ambientais e Saúde pela Pontifícia Universidade Católica de Goiás (PUC-GO), Goiânia-GO, Brasil.

3.Fisioterapeuta, Doutora, docente do Curso de Fisioterapia da Universidade Estadual de Goiás (UEG), Goiânia-GO, Brasil.
Endereço para correspondência: Cibelle Kayenne Martins Roberto Formiga Universidade Estadual de Goiás (UEG) Escola Superior de Educação Física e Fisioterapia de Goiás - ESEFFEGO Av. Anhanguera, $\mathrm{n}^{\circ} 1420$, Setor Vila Nova CEP 74705-010, Goiânia-GO, Brasil.

E-mail: cibellekayenne@gmail.com 


\section{INTRODUÇÃO}

A Paralisia Cerebral (PC) descreve um grupo de desordens permanentes do desenvolvimento do movimento e da postura, causando limitaçóes nas atividades, que são atribuídos a distúrbios não-progressivos ocorridos durante o desenvolvimento fetal ou no cérebro infantil. As alterações motoras da PC são frequentemente acompanhadas por distúrbios de sensação, percepção, cognição, comunicação, comportamento, epilepsia e problemas osteomusculares secundários ${ }^{1}$.

A incidência de PC no Brasil é de 2 a 3 crianças a cada 1.000 nascidas vivas, com a maior prevalência da forma espástica. E a incidência geral, incluindo todas as formas, em países desenvolvidos são de 7 crianças a cada 1.000 nascidas vivas ${ }^{2}$. A incidência nas formas moderadas e severa está entre 1,5 e 2,5 por 1.000 nascidas vivas em países desenvolvidos ${ }^{3}$.

A classificação da PC pode ser de acordo com a região acometida no cérebro, segundo o seu grau de comprometimento, pela distribuição topográfica da lesão e também pelas aquisiçôes da função motora grossa. Quanto ao tipo clínico pode ser: espástico, extrapiramidal, atáxico, hipotônico e misto ${ }^{4}$. Quanto à distribuição topográfica a Paralisia Cerebral pode ser classificada em hemiplegia, diplegia e quadriplegia ${ }^{1}$. A hemiplegia atinge os membros contra laterais ao lado do cérebro afetado. O crescimento do lado afetado do corpo é frequentemente menor em relação ao lado oposto ${ }^{5}$. $\mathrm{Na}$ diplegia espástica a criança apresenta problema motor bilateral dos quatros membros, sendo os membros inferiores mais acometi$\operatorname{dos}^{6}$. Enquanto a quadriplegia é clinicamente caracterizada por acometimento global. Devido ao comprometimento muscular global a marcha fica comprometida 5 .

A multiplicidade de sintomas motores, sensoriais e cognitivos dificulta frequentemente a identificação do impacto da Paralisia Cerebral nas habilidades funcionais, num universo que perpassa as esferas da saúde, educação e comunidade. O diagnóstico funcional da criança com PC e a mensuração da efetividade das intervençôes terapêuticas adotadas no seu desempenho são imprescindíveis para qualificação dos serviços prestados à essa população ${ }^{7}$.

A PC é uma condição clínica que gera alta prevalência de sequelas funcionais. As crianças com este diagnóstico podem apresentar diversos problemas nas ativida- des funcionais, no controle dos movimentos voluntários, o que pode prejudicar as suas capacidades diárias, bem como sobrecarregar cuidadores e terapeutas ${ }^{8}$. Ao avaliar habilidades funcionais de crianças, estudos sugerem que atitudes e expectativas dos pais e cuidadores possam exercer influência negativa ou positiva na performance e independência funcional das crianças com Paralisia Cerebral ${ }^{7,9-11}$. Desta forma, o presente estudo baseou-se na necessidade de entendimento da relação existente entre o desempenho nas habilidades funcionais na área de autocuidado, mobilidade e função social de crianças com PC e o nível de envolvimento/participação de cuidadores na realização destas atividades. Neste sentido, o estudo teve por objetivo verificar a associação entre o desempenho funcional e o nível de assistência do cuidador de crianças com PC.

\section{MÉTODO}

\section{Amostra}

Este estudo caracteriza-se como transversal e contou com uma amostra de 12 crianças com diagnóstico clínico de Paralisia Cerebral. Os critérios de inclusão foram: crianças classificadas como nível I e II do Sistema de Classificação da Função Motora Grossa (GMFCS - Gross Motor Function Classification System), de ambos os sexos, com idade entre 1 ano e 6 meses a 5 anos e 4 meses, frequentadoras o Setor Clínico da Associação Pestalozzi de Goiânia (Unidade Renascer).

O estudo foi realizado conforme as Diretrizes e Normas Reguladoras de Pesquisa Envolvendo Seres $\mathrm{Hu}$ manos (Resolução 196/1996 do Conselho Nacional de Saúde) e aprovado pelo Comitê de Ética em Pesquisa da Universidade Federal de Goiás (parecer nº0205/10).

\section{Procedimento}

Os pais/responsáveis leram e assinaram o Termo de Consentimento Livre e Esclarecido (TCLE). Para caracterização da amostra os mesmos responderam, através de entrevista, uma ficha de avaliação neurológica, elaborada pelas autoras, e um questionário de nível socioeconômico elaborado pela Associaçáo Brasileira de Empresas e Pesquisa (ABEP) - Critério de Classificação Econômica Brasil $^{12}$.

O Sistema de Classificação da Função Motora 
Grossa (GMFCS) usado na versão traduzida em português ${ }^{13}$ foi aplicado com o objetivo de classificar o nível da função motora das crianças com Paralisia Cerebral, como critério de inclusão e caracterização da amostra. É um instrumento designado em uma escala ordinal de cinco níveis, de maneira decrescente ${ }^{14-16}$.

No nível I do GMFCS, as crianças andam nos espaços internos e externos e sobem escadas sem limitaçôes. As crianças realizam habilidades motoras grossas, incluindo correr e pular, mas a velocidade, o equilíbrio e a coordenação estão reduzidos. No nível II, as crianças andam nos espaços internos e externos e sobem escadas segurando-se no corrimão, mas apresentam limitações ao andar em superfícies irregulares inclinadas e em espaços lotados ou restritos. As crianças, com maiores habilidades neste nível apresentam capacidade mínima para realizar habilidades motoras grossas como correr e pular ${ }^{13}$.

Após a autorização dos pais, as crianças foram avaliadas pelo Pediatric Evaluation of Disability Inventory (PEDI) através de entrevista estruturada com o cuidador. O referido instrumento objetiva avaliar o desenvolvimento de habilidades funcionais e o nível de independência no desempenho de atividades funcionais, no ambiente típico da criança, em três áreas: autocuidado, mobilidade e função social ${ }^{17}$. A escala de autocuidado abrange alimentação, higiene pessoal, uso do toalete, vestuário e controle esfincteriano. Os itens funcionais de mobilidade informam sobre transferências, locomoçáo em ambiente externo e interno, e uso de escadas. E a dimensão função social reflete as questôes relativas à comunicação, resolução de problemas, interação com colegas, entre outros ${ }^{18}$.

No presente estudo foram utilizadas as partes I e II do instrumento, a primeira refer-se ao desempenho funcional em atividades do dia-a-dia, a segunda parte diz respeito ao nível de assistência que o cuidador oferece à criança na execução das tarefas diárias. Na Parte I, a pontuação em escore é 1 (se a criança é capaz) ou 0 (se a criança não é capaz). $\mathrm{Na}$ Parte II, a pontuação em escala ordinal é 0 (necessidade de assistência total), 1 (necessidade máxima), 2 (necessidade média), 3 (necessidade mínima), 4 (supervisão) e 5 (sem necessidade - independente). Para as duas partes os escores são somados em cada área de função ${ }^{8}$. E para a pontuação geral, neste estudo foi utilizado o tipo de escore normativo (percentil de desempenho).
Neste teste, a independência da criança é inversamente avaliada através da quantidade de assistência que é fornecida pelo cuidador no desempenho de atividades funcionais ${ }^{17}$. Desta forma, quanto mais ajuda a criança receber de seu cuidador para realizar as tarefas funcionais, menor será a sua independência nestas tarefas ${ }^{19}$.

\section{Análise Estatística}

A análise dos dados foi realizada pelo programa Statistical Package for Social Science (SPSS) versão 14.0. Inicialmente, os dados de caracterização da amostra foram analisados através de estatística descritiva, incluindo as variáveis categóricas do perfil das crianças (frequência e porcentagem) e contínuas da pontuação da criança no PEDI (média, desvio padrão, valores mínimo e máximo). Em seguida, foi realizado teste de Correlação de Pearson para verificar a associação (coeficiente de correlação - r) entre o desempenho funcional e o nível de assistência do cuidador das crianças com Paralisia Cerebral. Em todas as análises realizadas foi considerado o $\mathrm{p}<0,05$.

\section{RESULTADOS}

Da amostra composta pelas 12 crianças, a idade variou de 1 ano e 6 meses (idade mínima) a 5 anos e 4 meses (idade máxima) com média de 3 anos e 2 meses e desvio padráo de 1 ano e 2 meses. Verifica-se que houve predomínio de crianças com PC de etiologia pré-natal (50\%), com hemiplegia (58\%), nível I do GMFCS (83\%), que não fazem uso de órteses (67\%), do sexo masculino (75\%) e classe socioeconômica C (75\%, Tabela 1).

A média dos escores normativos encontrada em cada área avaliada sugere que as crianças em estudo apresentaram atraso ou desempenho significativamente inferior (escore abaixo de 30) ao demonstrado por crianças de mesma faixa etária e com desenvolvimento típico, nas habilidades funcionais quanto à Mobilidade e na Assistência do Cuidador quanto à Mobilidade e Função Social. Nas habilidades de Autocuidado e Função Social e na Assistência do Cuidador no Autocuidado observa-se através das médias que o desempenho das crianças está dentro do intervalo de normalidade (escore entre $30 \mathrm{e}$ 70 ), o esperado para crianças de mesma faixa etária com desenvolvimento típico (Tabela 2).

As correlaçôes encontradas entre as áreas de habili- 


\begin{tabular}{|c|c|c|}
\hline & Frequência (f) & Porcentagem (\%) \\
\hline \multicolumn{3}{|l|}{ Idade } \\
\hline 1 ano e 6 meses & 2 & 16,7 \\
\hline 2 anos & 2 & 16,7 \\
\hline 2 anos e 6 meses & 1 & 8,3 \\
\hline 3 anos e 6 meses & 1 & 8,3 \\
\hline 3 anos e 7 meses & 1 & 8,3 \\
\hline 3 anos e 9 meses & 2 & 16,7 \\
\hline 4 anos & 2 & 16,7 \\
\hline 5 anos e 4 meses & 1 & 8,3 \\
\hline \multicolumn{3}{|l|}{ Etiologia da PC } \\
\hline Pé Natal & 6 & 50 \\
\hline Peri Natal & 4 & 33 \\
\hline Pós Natal & 2 & 17 \\
\hline \multicolumn{3}{|l|}{ Sexo } \\
\hline Feminino & 3 & 25 \\
\hline Masculino & 9 & 75 \\
\hline \multicolumn{3}{|c|}{ Classificaçáo da ABEP } \\
\hline Classe B1 e B2 & 2 & 17 \\
\hline Classe C & 9 & 75 \\
\hline Classe D & 1 & 8 \\
\hline \multicolumn{3}{|l|}{ Uso de Órtese } \\
\hline Sim & 4 & 33 \\
\hline Não & 8 & 67 \\
\hline \multicolumn{3}{|l|}{ Tipo de Órtese } \\
\hline Não usa & 8 & 67 \\
\hline Usa em MMII & 4 & 33 \\
\hline \multicolumn{3}{|l|}{ GMFCS } \\
\hline Nível I & 10 & 83 \\
\hline Nível II & 2 & 17 \\
\hline \multicolumn{3}{|l|}{ Classificaçáo da PC } \\
\hline Hemiplegia & 7 & 58 \\
\hline Diplegia & 5 & 42 \\
\hline
\end{tabular}

PC - Paralisia Cerebral; ABEP - Associação Brasileira de Empresas e Pesquisa; MMII - Membros Inferiores; GMFCS - Sistema de Classificação da Função Motora Grossa

dades funcionais mostraram que o desempenho funcional das crianças avaliadas na área de autocuidado teve uma associação positiva com as habilidades funcionais de mobilidade e de função social, indicando que quanto maior a funcionalidade nas atividades sociais e motoras, maior a capacidade da criança na realização de tarefas do autocuidado, tais como, vestir, alimentar, higiene pessoal. Observa-se também associação positiva entre as habilidades funcionais de mobilidade e função social, indicando que quanto maior a habilidade motora da criança, maior será seu desempenho nas atividades de função social (Tabela 3 ).

Quanto às correlaçóes encontradas entre as Habilidades Funcionais e Auxílio do Cuidador verificou-se que as habilidades funcionais de autocuidado tiveram associação positiva significativa com a assistência do cuidador nas áreas de autocuidado $(r=0,87 ; \mathrm{p}<0,001)$ e mobilidade 
Tabela 2

Resultados da pontuaçâo das crianças nas áreas avaliadas pelo escore normativo do PEDI ( $n=12)$

\begin{tabular}{ccccc}
\hline Variável & Máximo & Mínimo & Média & DP \\
\hline HFAC & 70,5 & abaixo de 10 & 30,4 & 21,17 \\
HFM & 63 & abaixo de 10 & 21,19 & 19,15 \\
HFFS & 75,2 & abaixo de 10 & 34,8 & 17,63 \\
ACAC & 48,5 & abaixo de 10 & 30,67 & 13,5 \\
ACM & 60,3 & abaixo de 10 & 23,69 & 18,6 \\
ACFS & 42,9 & abaixo de 10 & 26,33 & 12,43 \\
\hline
\end{tabular}

DP - Desvio Padrão; HFAC - Habilidades Funcionais em Autocuidado;

HFM - Habilidades Funcionais em Mobilidade; HFFS - Habilidades Funcionais em Função Social;

ACAC - Assistência do Cuidador em Autocuidado; ACM - Assistência do Cuidador em Mobilidade;

ACFS - Assistência do Cuidador em Função Social

Tabela 3

Resultados das associaçôes entre Habilidades Funcionais e Assistência do Cuidador (n=12)

\begin{tabular}{lccc}
\hline \multicolumn{2}{l}{ Variáveis Correlacionadas } & Coeficiente de Correlaçáo (r) & Valor de $\mathbf{p}^{*}$ \\
\hline HFAC & HFM & 0,8 & 0,001 \\
HFAC & HFFS & 0,75 & 0,005 \\
HFFS & HFM & 0,57 & 0,048 \\
HFAC & ACAC & 0,87 & $<0,001$ \\
HFAC & ACM & 0,61 & 0,033 \\
HFM & ACM & 0,78 & 0,003 \\
\hline
\end{tabular}

*Correlaçáo de Pearson, $\mathrm{p}<0,05$

HFAC - Habilidades Funcionais em Autocuidado; HFM - Habilidades Funcionais em Mobilidade;

HFFS - Habilidades Funcionais em Função Social; ACAC - Assistência do Cuidador em Autocuidado;

ACM - Assistência do Cuidador em Mobilidade

$(\mathrm{r}=0,61 ; \mathrm{p}=0,033)$, indicando que quanto maior for o resultado na habilidade de autocuidado, maior será o escore de assistência do cuidador nas habilidades de autocuidado e mobilidade e, consequentemente, maior será o nível de independência da criança nestas áreas. As habilidades funcionais de mobilidade apresentaram associação significativa com a assistência do cuidador na mobilidade ( $r=0,78$; $\mathrm{p}=0,003)$, indicando que, quanto maior a capacidade da criança nas atividades funcionais de mobilidade, tais como, transferências e locomoção em ambientes, menor é a quantidade de ajuda fornecida pelo cuidador e maior será sua independência quanto à mobilidade (Tabela 3).

\section{DISCUSSÃO}

Os resultados obtidos no presente estudo verificaram associação entre o nível de desempenho funcional e a assistência do cuidador em crianças com paralisia cerebral. O escore padronizado normativo do PEDI informa sobre o desempenho esperado de crianças da mesma faixa etária, com desenvolvimento típico. Escore normativo de magnitude entre 30 e 70 é considerado dentro do intervalo de normalidade, esperado para crianças brasileiras com desenvolvimento típico, de mesma faixa etária. Escores normativos inferiores a 30 ilustram atraso ou desempenho significativamente inferior ao demonstrado por crianças de mesma faixa etária. Escore normativo superior a 70 sugere desempenho significativamente superior àquelas crianças com desenvolvimento típico e de mesma faixa etária ${ }^{19}$.

Ao avaliar as habilidades funcionais e a necessidade de auxílio do cuidador em 7 crianças com Paralisia Cerebral, quadro motor de diparesia e tetraparesia, com idade até 18 meses, os resultados obtidos ${ }^{20}$ mostram que a maior dificuldade apresentada por elas está na área de mobilidade, tanto nas habilidades funcionais como no auxílio do cuidador. Desta forma, há necessidade de maior assistência do cuidador em movimentos e posturas que facilitem a locomoção e transferência das crianças. 
O presente estudo obteve resultados semelhantes ao dos autores, embora tenha utilizado amostra de crianças com Paralisia Cerebral com diferente quadro motor e faixa etária. A média dos escores normativos encontrados na área de mobilidade foram as menores encontradas nas Habilidades Funcionais e no Auxílio do Cuidador.

Pesquisadores $^{8}$ utilizaram o PEDI para investigar 10 crianças com Paralisia Cerebral diparética espástica, com idade entre 4 e 5 anos, e compararam com 10 crianças com desenvolvimento típico na mesma faixa etária. Análises comparativas nas 3 áreas de desempenho ocupacional mostraram diferença significativa nas áreas de autocuidado e mobilidade, e não foi observada diferença significativa entre os grupos na área de função social. Estes resultados revelaram que crianças com PC na faixa etária investigada apresentam desempenho inferior em áreas que envolvem habilidades de autocuidado e mobilidade. No presente estudo os resultados foram semelhantes em que as crianças tiveram desempenho inferior na área de mobilidade. Portanto, na área de autocuidado, ao considerar que a amostra é predominantemente composta por crianças hemiplégicas, de nível I, com faixa etária média menor do que a referenciada, é possível que estas consigam realizar atividades funcionais de autocuidado como o esperado por crianças da mesma faixa etária, com desenvolvimento típico.

Ao correlacionar as habilidades funcionais referidas pelo cuidador e a assistência fornecida à criança com paralisia cerebral do tipo diparesia espástica, estudo ${ }^{7}$ indicou relação linear positiva significativa nas áreas de mobilidade e função social. Entretanto, o mesmo tipo de relação não se verificou na área do autocuidado.

Os resultados encontrados no atual estudo assemelham-se à outro ${ }^{7}$ quanto à ocorrência de correlação nas Habilidades Funcionais e Assistência do Cuidador na área de Mobilidade. Porém, diferencia-se quando não observa correlação significativamente positiva quanto à área de função social, e quando observa uma correlação significativamente positiva quanto a área de autocuidado, apresentando alto coeficiente de correlação nesta área. Vale considerar ainda que o estudo referenciado foi analisado através do escore bruto obtido no PEDI com crianças diparéticas, não mencionado o nível motor, e a idade média das crianças foi de 6 anos. Os próprios auto- res citam que a não observação de correlação na área de autocuidado foi um resultado diferente dos encontrados na literatura utilizando o PEDI.

Levando em consideração as implicações motoras, cognitivas e sensoriais da Paralisia Cerebral, este estudo verificou na amostra pesquisada que a Assistência do Cuidador está diretamente associada ao desempenho da criança nas Habilidades Funcionais de Autocuidado e Mobilidade. Sendo a independência da criança inversamente avaliada através da quantidade de assistência que é fornecida pelo cuidador ${ }^{17}$, pode-se dizer que o nível de independência da criança também está associado ao seu desempenho. E, portanto, quanto maior o nível de desempenho nas habilidades funcionais das crianças com PC nas áreas de autocuidado e mobilidade, menor é a quantidade de auxílio do cuidador fornecida à criança durante estas atividades funcionais. Verificou-se também que quanto menor for a quantidade de auxílio fornecido pelo cuidador nas atividades de mobilidade, mais independentes as crianças se apresentarão nesta área e desempenharão melhor as atividades funcionais de autocuidado.

Os conhecimentos mais aprofundados acerca do desempenho funcional dessas crianças e do nível de assistência dos cuidadores são fundamentais para desenvolver estratégias em saúde e educação para as crianças e suas famílias, para um melhor direcionamento dos profissionais quanto ao uso de avaliaçóes, planejamento de tratamento, metas, envolvimento e orientaçóes à cuidadores, contribuindo para qualidade de vida das crianças e qualificação dos serviços prestados.

\section{CONCLUSÃO}

O instrumento de avaliação PEDI demostrou-se válido e confiável para avaliação do desempenho funcional e da assistência do cuidador em crianças com Paralisia Cerebral, sendo possível verificar suas correlações.

Neste estudo, foi observada correlação positiva entre a assistência fornecida pelo cuidador e o desempenho funcional das crianças na área do autocuidado e da mobilidade. Também se verificou correlação significativa no nível de independência da criança quanto à mobilidade e o desempenho da mesma nas atividades de autocuidado. Frente a estes resultados ressalta-se a necessidade dos profissionais envolverem de forma mais intensa os cuidado- 
res nos tratamentos e atividades direcionadas às crianças com Paralisia Cerebral para que a família tenha melhor compreensão das potencialidades e capacidades funcionais de seus filhos e busquem através de atitudes apoiar a autonomia nas atividades funcionais de autocuidado e mobilidade de sua criança.

\section{REFERÊNCIAS}

1.Rosenbaum P, Paneth N, Leviton A, Goldstein M, Bax M. A report: the definition and classification of cerebral palsy April 2006. Dev Med Child Neurol Suppl 2007;109:8-14.

2.Allegretti KMG, Kanashiro MS, Monteiro VC, Borges HC, Fontes SV. Os efeitos do treino de equilíbrio em crianças com paralisia cerebral diparética espástica. Rev Neurocienc 2007;15(2):108-13.

3.Leite JMRS, Prado GF. Paralisia cerebral: aspectos fisioterapêuticos e clínicos. Rev Neurocienc 2004;12(1):41-5.

4.Graham HK. Classifying cerebral palsy. J Pediatr Orthop 2005;25(1):127-8. http://dx.doi.org/10.1097/00004694-200501000-00026

http://dx.doi.org/10.1097/01241398-200501000-00026

5.Lima CLA, Fonseca LF. Paralisia Cerebral. Rio de Janeiro: Guanabara Koogan; 2004, 492p.

6.Bax M, Flodmark O, Tydeman C. From syndrome toward disease. Department of Paediatrics (4th Floor), Chelsea \& Westminster Hospital, Fulham Rd, London, UK. Dev Med Child Neurol 2007;49:1-44.

7.Oliveira MC, Cordani LK. Correlação entre habilidades funcionais referidas pelo cuidador e assistência fornecida a crianças com paralisia cerebral. Arq Bras Paralis Cereb 2004;1:24-9.

8.Allegretti ALC, Mancini MC, Schwartzman JS. Estudo do desempenho funcional de crianças com paralisia cerebral diparética espástica utilizando o Pediatric Evalution of Disability Inventory (PEDI). Arq Bras Paralis Cereb 2004;1(1):35-40.

9.Mancini MC, Fiuza PM, Rebelo JM, Magalhães LC, Coelho ZAC, Paixão ML, et al. Comparaçáo do desempenho de atividades funcionais em crianças com desenvolvimento normal e crianças com paralisia cerebral. Arq Neuro-Psiquiatr 2002;60(2B):446-52.
http://dx.doi.org/10.1590/S0004-282X2002000300020

10.Mancini MC, Alves ACM, Schaper C, Figueiredo EM, Sampaio RF, Coelho ZA, et al. Gravidade da paralisia cerebral e desempenho funcional. Rev Bras Fisioter 2004;8(3):253-60.

11. Oliveira AKC, Alves ACJ. Desempenho funcional de crianças com paralisia cerebral e a percepção dos cuidadores.[monografia]. São Carlos: Universidade Federal de São Carlos; 2010, 50p. Especialização em Intervenção em Neuropediatria.

12.Critério de classificação econômica Brasil 2010. São Paulo. Associação Brasileira de Empresas de Pesquisa (ABEP). [atualizado em: 2012; acessado em: 06/2012] Disponível em: http://www.abep.org/novo/Content. aspx?ContentID=301.

13. Hiratuka E, Matsukura TS, Pfeifer LI: Cross-cultural adaptation of the Gross Motor Function Classification System into Brazilian-Portuguese (GMFCS). Rev Bras Fisioter 2010;14(6):537-44.

http://dx.doi.org/10.1590/S1413-35552010000600013

14.Palisano R, Rosenbaum P, Walter S, Russell D, Wood E, Galuppi B.. Development and reliability of a system to classify gross motor function in children with cerebral palsy. Dev Med Child Neurol 1997;39:214-23.

http://dx.doi.org/10.1111/j.1469-8749.1997.tb07414.x

15.Morris C, Kurinczuk JJ, Fitzpatrick R, Rosenbaum PL. Do the abilities of children with cerebral palsy explain their activities and participation? Dev Med Child Neurol 2006;48(12):954-61.

http://dx.doi.org/10.1111/j.1469-8749.2006.tb01265.x

16.Morris C, Kurinczuk JJ, Fitzpatrick R, Rosenbaum PL. Reliability of the manual ability classification system for children with cerebral palsy. Dev Med Child Neurol 2006b,48(12):950-3.

http://dx.doi.org/10.1111/j.1469-8749.2006.tb01264.x

17. Chagas PSC, Mancini MC. Testes padronizados utilizados na avaliação da criança portadora de paralisia cerebral. In: Lima CLF, Fonseca LF. Paralisia cerebral: neurologia, ortopedia, reabilitaçâo. Rio de Janeiro: Guanabara Koogan, 2004, p.318-38.

18.Chagas PSC, Defilipo EC, Lemos RA, Mancini MC, Frônio JS, Carvalho RM. Classificaçấo da funçấo motora e do desempenho funcional de crianças com paralisia cerebral. Rev Bras Fisioter 2008;12(5):409-16.

http://dx.doi.org/10.1590/S1413-35552008000500011

19.Mancini MC. Inventário de Avaliação Pediátrica de Incapacidade (PEDI), Manual da Versão Brasileira Adaptada. Belo Horizonte: UFMG, 2005, 193p. 20. Herrero D, Monteiro CBM. Verificação das habilidades funcionais e necessidades de auxílio do cuidador em crianças com paralisia cerebral nos primeiros meses de vida. Rev Bras Crescimento Desenvolv Hum 2008;18(2):163-9. 